\title{
Défavorisation, accès aux aliments et équilibre alimentaire à Saskatoon (Saskatchewan)
}

\author{
J. Cushon, Ph. D. (1); T. Creighton, M. Sc. SIG (1); T. Kershaw, MHP (1); J. Marko, MHP (1); T. Markham, \\ B. Sc. Économie domestique (2)
}

Cet article a fait l'objet d'une évaluation par les pairs.

\section{Résumé}

Introduction : Nous avons étudié l'accès aux aliments et l'équilibre alimentaire à Saskatoon (Saskatchewan, Canada) en lien avec la défavorisation matérielle et sociale.

Méthodologie : Nous avons déterminé l'emplacement de tous les grands supermarchés et des restaurants-minute à Saskatoon. L'indice d'accessibilité aux supermarchés et celui de l'équilibre alimentaire ont été comparés aux indices de défavorisation matérielle et sociale afin de déterminer s'il y avait une association importante.

Résultats : Les endroits où il y a le moins de supermarchés sont les quartiers situés à l'ouest de la rivière Saskatchewan Sud ainsi que la banlieue entourant la ville. Les quartiers de l'ouest de la rivière sont les plus défavorisés de la ville. Le ratio moyen de l'équilibre alimentaire de Saskatoon est de 2,3 , ce qui indique une prédominance de l'accès aux restaurants-minute. Cependant, nous n'avons pas trouvé de schéma ou de gradient socioéconomique clairs pour plusieurs des indicateurs.

Conclusion : L'étude souligne l'importance des études contextuelles sur l'accès aux aliments. L'étude met également en lumière un certain nombre d'autres questions qui devraient être étudiées à Saskatoon, comme les habitudes alimentaires individuelles, la mobilité, l'évolution dans le temps de l'accès aux aliments, l'accès économique ainsi que les interventions susceptibles d'améliorer l'accès aux aliments dans la ville.

Mots-clés : accès aux aliments, santé, défavorisation, statut socioéconomique

\section{Introduction}

L'accès à des aliments sains et nutritifs est associé à des résultats sur la santé sur le plan écologique ${ }^{1-4}$. L'étude de l'accès aux aliments dans certaines zones géographiques présente donc de plus en plus d'intérêt, plus particulièrement en ce qui a trait aux écarts en matière d'accès compte tenu de facteurs comme le statut socioéconomique (SSE).

\section{Accès aux aliments et santé}

L'accès aux aliments est habituellement mesuré en termes de proximité à certains types de magasins d'alimentation ${ }^{5}$. Les grands supermarchés fournissent généralement une plus grande variété d'aliments sains à des prix plus bas que les petites épiceries ou les dépanneurs ${ }^{6-13}$. Certaines recherches montrent que, comparativement aux résidents qui vivent plus loin, ceux qui vivent près des super- marchés sont consomment davantage de fruits et légumes et, dans l'ensemble, ont des régimes alimentaires plus sains et des taux d'obésité plus faibles ${ }^{6,14-19}$.

Par ailleurs, les restaurants-minute vendent habituellement des aliments transformés et peu nutritifs, qui sont hypercaloriques et à forte teneur en sodium $^{20}$. Selon certaines études, le fait de résider près de restaurants-minute est associé à des régimes alimentaires moins sains, à l'obésité et à une moins bonne santé, avec des maladies telles que le diabète et les maladies du cœur ${ }^{6,16,21-24}$. Cependant, selon d'autres études, il n'existe pas de lien entre la proximité de restaurants-minute et les effets sur le régime alimentaire et la santée ${ }^{25-27}$.

\section{Équilibre alimentaire et santé}

L'équilibre alimentaire se mesure par le ratio entre la proximité des supermarchés (et donc l'accès à des aliments sains) et la proximité des restaurants-minute (et donc, l'accès à des aliments malsains). Il a été déterminé que l'équilibre alimentaire est associé aux résultats sur la santé ${ }^{28,29}$. Par exemple, une étude menée à Edmonton (Alberta) a montré que l'équilibre alimentaire était significativement associé à l'obésité : moins un endroit était " équilibré », plus il était probable qu'un résident soit obèse ${ }^{29}$.

\section{Accès aux aliments et statut socioéconomique}

Le gradient socioéconomique du régime alimentaire, selon lequel les groupes d'un 
SSE élevé consomment des aliments plus sains que ceux d'un SSE moins élevé, existe à l'échelle internationale comme nationale ${ }^{30}$. L'un de ses facteurs explicatifs est l'accès : les résidents de quartiers défavorisés ont un accès moindre à des aliments sains que les résidents de quartiers plus riches ${ }^{31}$. Les résidents à faible revenu n'ont pas toujours accès à un véhicule, ce qui amplifie la difficulté d'accès aux aliments ${ }^{13,24,32}$. Selon une étude à méthodes mixtes menée à Saskatoon (Saskatchewan), les principaux facteurs ayant une influence sur l'achat des aliments étaient la possession d'un véhicule, le revenu et la facilité d'accès ${ }^{33}$.

Des études américaines ont conclu que l'accès à des aliments sains est généralement plus difficile dans les quartiers défavorisés ${ }^{21,34}$. Toutefois, des études empiriques menées dans d'autres pays industrialisés tirent des conclusions mitigées. Des études menées au Canada, en Australie et en Écosse ne tirent pas de conclusion dans ce sens, certaines concluant même que les quartiers à faible revenu offraient un meilleur accès à des aliments sains que les quartiers mieux nantis $8,12,20,35$.

\section{Déserts alimentaires}

$\mathrm{Au}$ cours de la dernière décennie, les travaux de recherche sur l'accès aux aliments ont porté de plus en plus sur les déserts alimentaires. Selon le département de l'Agriculture des États-Unis, un désert alimentaire est un secteur de recensement à faible revenu où une proportion ou un nombre important de résidents ont difficilement accès à un supermarché ou à une grande épicerie ${ }^{36}$. Bien que des recherches américaines aient conclu sans équivoque à l'existence de déserts alimentaires, les résultats concernant les déserts alimentaires au Canada sont moins concluants ${ }^{34}$. En fait, une étude menée à Montréal (Québec) a conclu que cette ville compte très peu de déserts alimentaires et que les résidents à faible revenu y avaient un meilleur accès à des supermarchés que les autres résidents ${ }^{7}$. Selon une autre étude, les résidents à faible revenu d'Edmonton, en Alberta, auraient en moyenne un meilleur accès aux supermarchés ${ }^{10}$ Cependant, les résidents les plus pauvres étaient 2,3 fois plus susceptibles d'habiter à 5-10 minutes à pied d'un restaurantminute que ceux qui vivaient dans des quartiers mieux nantis ${ }^{37}$. Ces conclusions s'apparentent à celles des études américaines $^{38-40}$. Selon une étude menée à London, en Ontario, certains quartiers à faible revenu constituent des déserts alimentaires même si dans certains quartiers mieux nantis l'accès à des supermarchés est difficile $\mathrm{e}^{41}$. Cela laisse croire que la recherche sur l'accès aux aliments doit se faire au cas par cas pour guider les politiques et les pratiques locales ${ }^{31,34}$.

Le but de notre étude était de répondre aux questions de recherche suivantes :

1) Comment l'accès aux aliments et l'équilibre alimentaire à Saskatoon varient-ils en lien avec la défavorisation du quartier?

2) À Saskatoon, l'accès aux aliments et l'équilibre alimentaire suivent-ils un gradient socioéconomique?

\section{Méthodologie}

\section{Magasins d'alimentation et restaurants- minute}

Nous avons défini les supermarchés comme des épiceries de détail appartenant à de grandes chaînes et offrant généralement une plus grande variété de produits sains à un prix moindre que les supermarchés plus petits et indépendants ou que les autres magasins d'alimentation (au moment de l'étude, les magasins Walmart à Saskatoon n'offraient pas une grande variété d'aliments) $)^{7,42}$. Afin de trouver l'emplacement de tous les supermarchés à Saskatoon et pour démontrer l'accès aux aliments sains $(n=22)$, nous avons utilisé plusieurs sources de données pour 2007 et 2008, en particulier l'annuaire téléphonique, des sites Web d'entreprises et des renseignements locaux. Les emplacements ont été vérifiés à l'aide d'un système de localisation GPS, de Google Earth et d'adresses de la Ville de Saskatoon. Tous les emplacements ont été géocodés dans une base de données.
Nous avons défini les restaurants-minute comme des restaurants où il n'y a pas de serveurs et où les clients paient leurs aliments avant de les recevoir, puis les emportent eux-mêmes à leur table ou à l'extérieur de l'établissement ${ }^{43}$. Nous avons déterminé l'emplacement de tous les restaurants-minute, de toutes les aires de restauration dans les centres commerciaux et de tous les dépanneurs qui servent des repas chauds $(n=120)$ en utilisant des données fournies par le service de la sécurité des collectivités (Safe Communities Department) de la région sanitaire de Saskatoon pour août 2008. Les emplacements ont été géocodés de la même manière que l'emplacement des supermarchés.

\section{Défavorisation}

Nous avons déterminé la défavorisation des quartiers à l'aide de l'indice de défavorisation mis au point par l'Institut national de santé publique du Québec (INSPQ) ${ }^{44}$. Cet indice permet de mesurer la défavorisation selon les aires de diffusion (AD), soit les plus petites aires utilisées pour le recensement canadien ${ }^{45}$. Les $\mathrm{AD}$ sont composées d'un ou de plusieurs îlots limitrophes regroupant de 400 à 700 résidents environ. L'indice de défavorisation comporte deux composantes : la défavorisation sociale et la défavorisation matérielle. La défavorisation sociale comprend trois variables : la proportion de familles monoparentales, la proportion de résidents vivant seuls et l'état matrimonial. La défavorisation matérielle est mesurée à l'aide des variables suivantes : le niveau de scolarité, le revenu moyen et la situation professionnelle. L'INSPQ nous a fourni pour cette étude les résultats relatifs aux $\mathrm{AD}$ de Saskatoon pour ce qui est de la défavorisation matérielle et de la défavorisation sociale. Les résultats relatifs aux $\mathrm{AD}$ ont été calculés en fonction du recensement canadien de 2006. Nous avons attribué aux $\mathrm{AD}$ les quintiles de défavorisation, chacun d'entre eux représentant environ $20 \%$ de la population de Saskatoon. Les figures 1 et 2 illustrent la défavorisation matérielle et la défavorisation sociale à Saskatoon. La rivière Saskatchewan Sud, 
FIGURE 1

Défavorisation matérielle, Saskatoon (Saskatchewan)

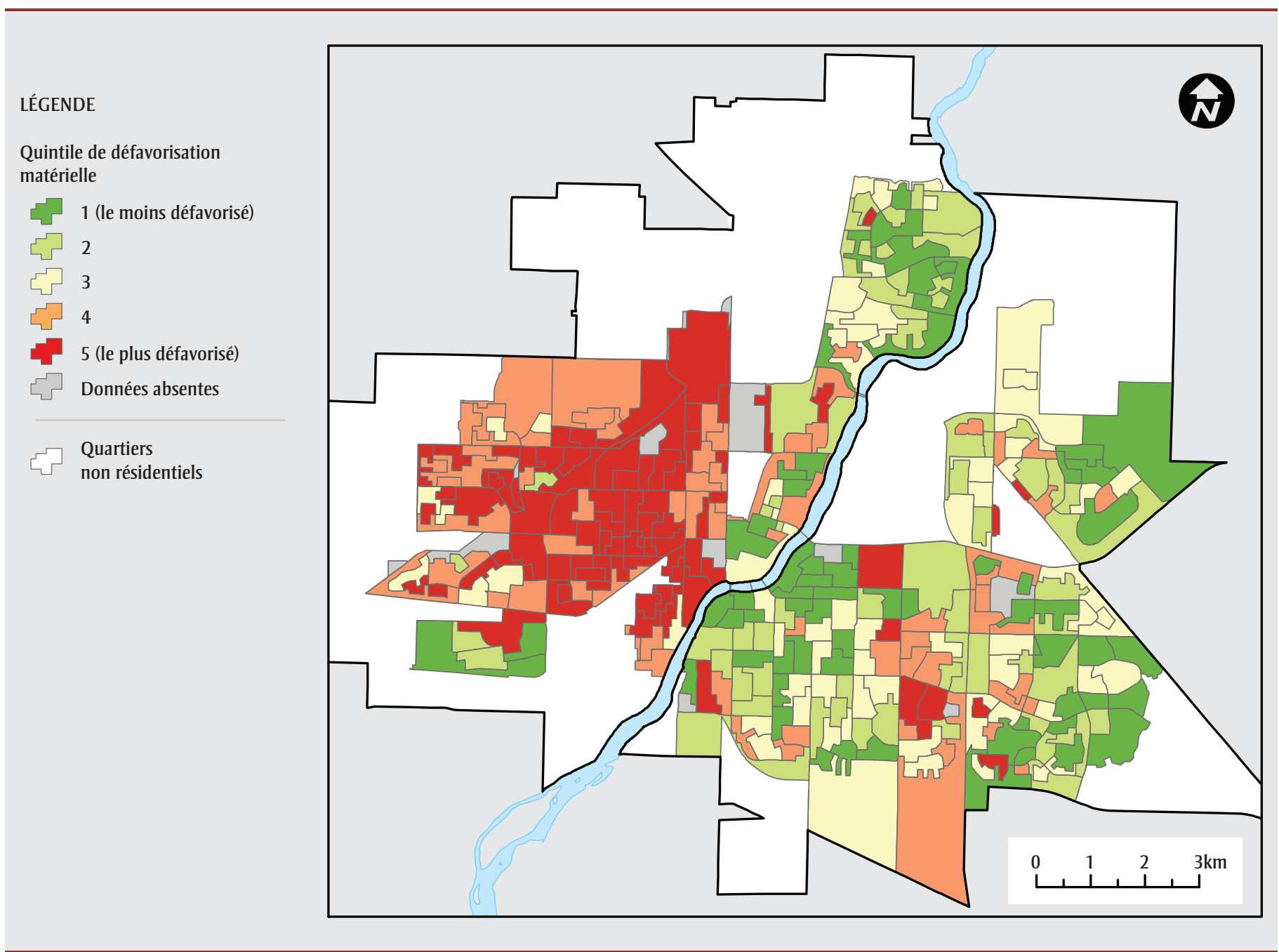

qui traverse Saskatoon, sépare la ville en deux, l'est et l'ouest.

\section{Accès aux aliments et équilibre alimentaire}

En nous fondant sur la méthodologie utilisée par Apparicio et coll. $^{7}$ pour Montréal (Québec), nous avons utilisé trois mesures pour déterminer le niveau d'accès aux supermarchés : proximité, diversité et variété. Ces mesures ont toutes été calculées à l'échelle des îlots urbains. Nous avons calculé la proximité en mesurant la distance euclidienne (c.-à-d. en ligne droite) entre le centroïde (c.-à-d. le centre géographique) de chaque îlot urbain et celui de chaque supermarché. Nous avons défini la diversité comme étant le nombre moyen de supermarchés situés à $1 \mathrm{~km}$ ou moins (c.-à-d. à distance de marche ${ }^{7}$ ) du centroïde de chaque îlot. La variété a été définie comme étant la distance moyenne entre le centroïde de chaque îlot et les trois supermarchés de chaînes différentes les plus proches. Les résidents qui vivent près de plusieurs chaînes différentes disposent en effet du plus grand choix de marques et de prix ${ }^{7}$.

Nous avons combiné les mesures de la proximité, de la diversité et de la variété afin de créer un indice d'accessibilité aux supermarchés. Il s'agit d'une approche novatrice en ce qui concerne la mesure de l'accessibilité aux aliments. Les résultats liés à la proximité ont été classés en tertiles en fonction des résultats relatifs à l'îlot. Si un îlot se classait dans le pire tertile en ce qui concerne la proximité, il obtenait une note de 1 , sinon il obtenait une note de 0 . Les résultats relatifs à la variété ont également été classés dans des tertiles, et les îlots ont été notés de la même manière que pour la proximité. Quant à la mesure de la diversité, un point supplémentaire était ajouté si un îlot se trouvait à plus d'1 km d'un supermarché. Nous avons additionné les résultats de chaque îlot pour produire un indice, la note maximale de 3 étant attribuée aux îlots ayant l'accès le plus faible à des supermarchés.

En divisant la distance minimale d'un supermarché par la distance minimale d'un restaurant-minute, nous avons obtenu un indice de l'équilibre alimentaire pour chaque îlot. Un indice d'équilibre alimentaire de 1 indique que les restaurants-minute et les supermarchés sont à 
FIGURE 2

Défavorisation sociale, Saskatoon (Saskatchewan)

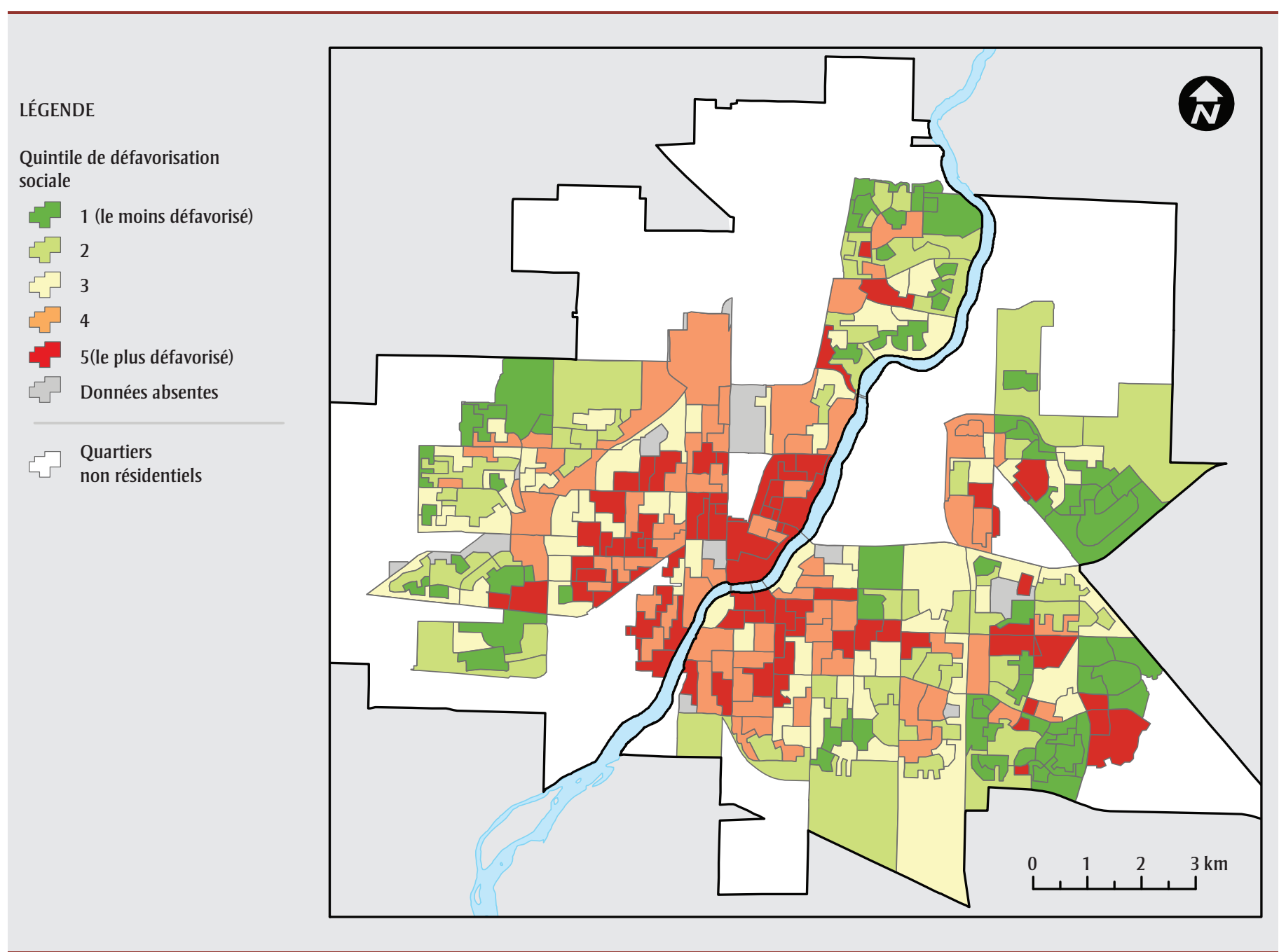

une distance égale du centroïde de l'îlot. Les quartiers dont l'indice de l'équilibre alimentaire est inférieur à 1, parce que le supermarché le plus proche se trouve plus près que le restaurant-minute le plus proche, ont été considérés comme plus " équilibrés » ${ }^{23}$.

Enfin, comme nos données n’obéissaient pas à une distribution normale, nous avons examiné les relations entre l'accès aux aliments, l'équilibre alimentaire, la défavorisation matérielle et la défavorisation sociale à l'aide des tests non paramétriques de Kruskal-Wallis. Nous avons eu recours ensuite aux tests U de MannWhitney puis à la correction de Bonferroni pour déterminer s'il existait d'importantes différences entre les quintiles de défavorisation.
Nous avons également tenté de cerner les secteurs préoccupants quant à l'accès aux aliments et à l'équilibre alimentaire. Ces secteurs étaient les quartiers défavorisés (quartiers se classant dans le cinquième quintile relativement à la défavorisation matérielle ou à la défavorisation sociale) avec accès difficile à des supermarchés (indice d'accessibilité de 3 ) et note pour l'équilibre alimentaire favorisant les restaurants-minute (indice d'équilibre alimentaire de 2 ou plus).

\section{Résultats}

\section{Supermarchés}

\section{Proximité}

Près de la moitié (45 \%) de la population vivait à $1 \mathrm{~km}$ ou moins d'un supermarché.
La distance moyenne entre les îlots résidentiels et le supermarché le plus proche était de 1094 mètres (figure 3; renseignements supplémentaires fournis sur demande).

La proximité variait fortement en fonction de la défavorisation matérielle (tableau 1; renseignements supplémentaires fournis sur demande) et de la défavorisation sociale (tableau 2; renseignements supplémentaires fournis sur demande). Par exemple, en ce qui concerne la défavorisation matérielle, les îlots les plus défavorisés (cinquième quintile) se trouvaient beaucoup plus loin du supermarché le plus proche que les îlots les moins défavorisés (premier quintile). La proximité diminuait généralement lorsque la défavorisation augmentait. 
FIGURE 3

Proximité du supermarché le plus proche, Saskatoon (Saskatchewan)

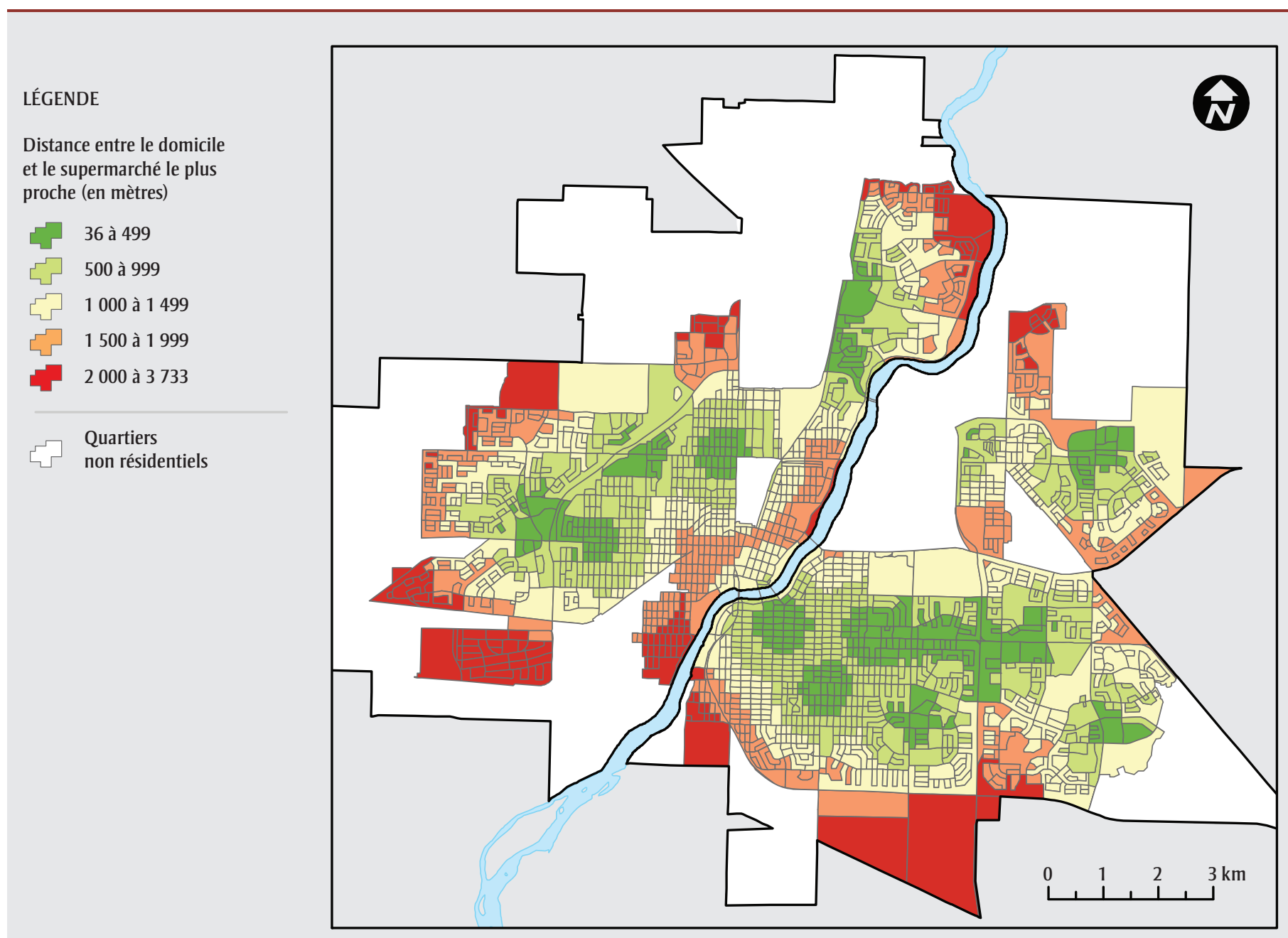

Cependant, en ce qui a trait à la défavorisation sociale, nous avons constaté que les îlots les moins défavorisés (premier quintile) se trouvaient beaucoup plus loin du supermarché le plus proche que ceux des autres quintiles. La proximité augmentait à mesure que la défavorisation diminuait, même si les troisième et cinquième quintiles étaient très similaires à cet égard.

\section{Diversité}

À Saskatoon, le nombre moyen relatif à la diversité était de 0,79 , c'est-à-dire que le résident moyen ne disposait pas de supermarché à distance de marche (1 km) (figure 4, renseignements supplémentaires fournis sur demande). La diversité était plutôt faible dans bon nombre de secteurs de la ville, surtout dans les secteurs défavorisés sur les plans matériel et social situés à l'ouest de la rivière Saskatchewan Sud et dans ceux en périphérie de la ville, là où la défavorisation était mixte (c.-à-d. que les îlots de ces secteurs se classaient dans tous les quintiles).

Nous avons également conclu que la diversité variait beaucoup en fonction de la défavorisation matérielle (tableau 1; renseignements supplémentaires fournis sur demande) et de la défavorisation sociale (tableau 2; renseignements supplémentaires fournis sur demande). La diversité était généralement moindre là où la défavorisation matérielle était plus grande, même si les îlots du deuxième quintile ne reflétaient pas cette tendance et que des îlots des deuxième et cinquième quintiles bénéficiaient d'une diversité moindre.
En ce qui a trait à la défavorisation sociale, les îlots les moins défavorisés (premier quintile) présentaient la diversité la plus faible de tous les quintiles.

\section{Variété}

La distance moyenne pour se rendre aux trois supermarchés de chaînes différentes les plus proches (figure 5) était de 1657 mètres. Comme pour la proximité et la diversité, la variété était considérablement moindre dans les secteurs les plus défavorisés situés à l'ouest de la rivière Saskatchewan Sud et dans les banlieues en périphérie de la ville, où la défavorisation était mixte.

La variété fluctuait beaucoup en fonction de la défavorisation matérielle et de la défavorisation sociale. En ce qui concerne la défavorisation matérielle, les îlots du 
TABLEAU 1

Statistiques relatives à l'accès aux aliments et à l'équilibre alimentaire par quintile de défavorisation matérielle, 2006, Saskatoon (Saskatchewan)

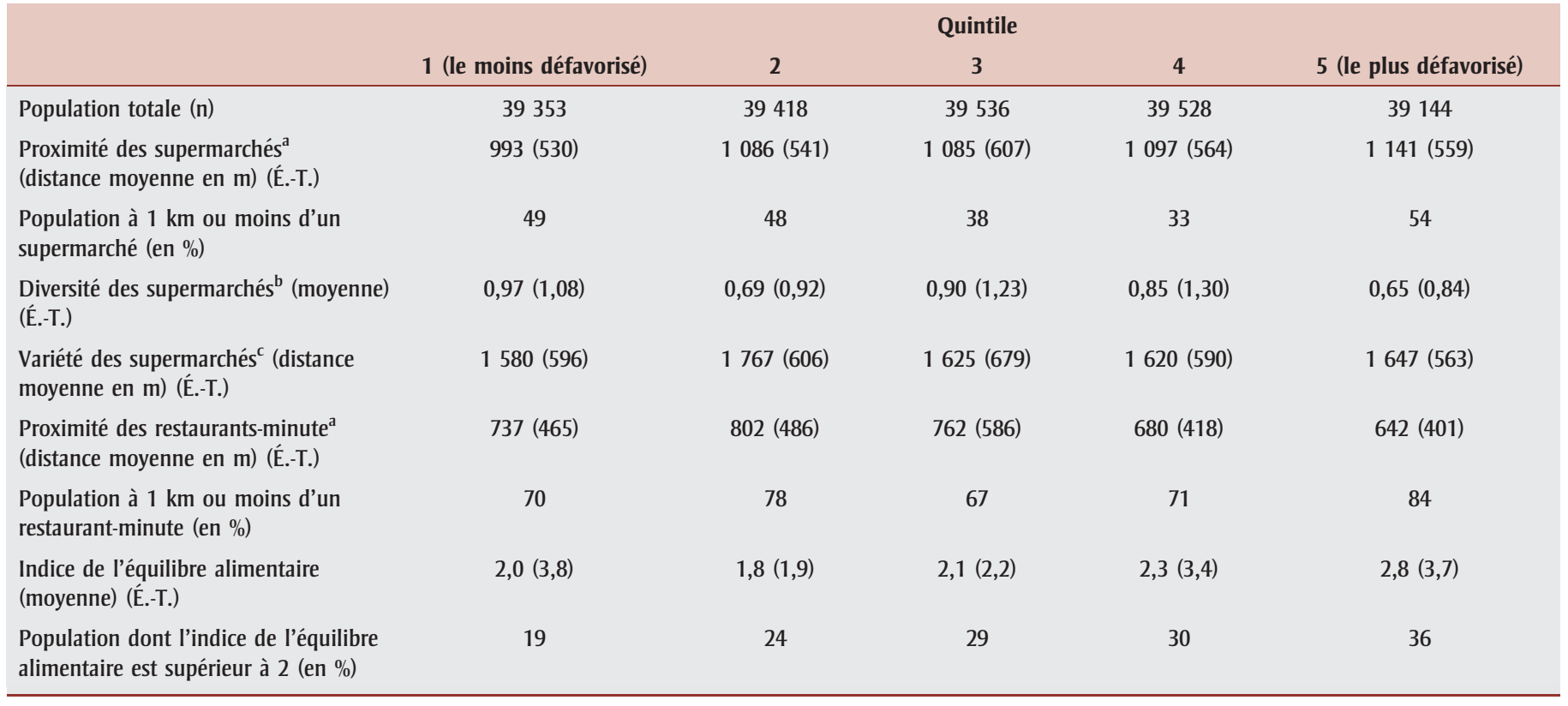

Abréviation : É.-T., écart-type.

${ }^{a}$ La proximité est définie comme étant la distance euclidienne (c.-à-d. en ligne droite) entre le centroïde (centre géographique) de chaque îlot urbain et chaque supermarché.

${ }^{\text {b }}$ La diversité est définie comme étant le nombre moyen de supermarchés à $1 \mathrm{~km}$ ou moins du centroïde de chaque îlot.

c La variété est définie comme étant la distance moyenne entre le centroïde de chaque îlot et les trois supermarchés de chaînes différentes les plus proche.

TABLEAU 2

Statistiques relatives à l'accès aux aliments et à l'équilibre alimentaire par quintile de défavorisation sociale, 2006, Saskatoon (Saskatchewan)

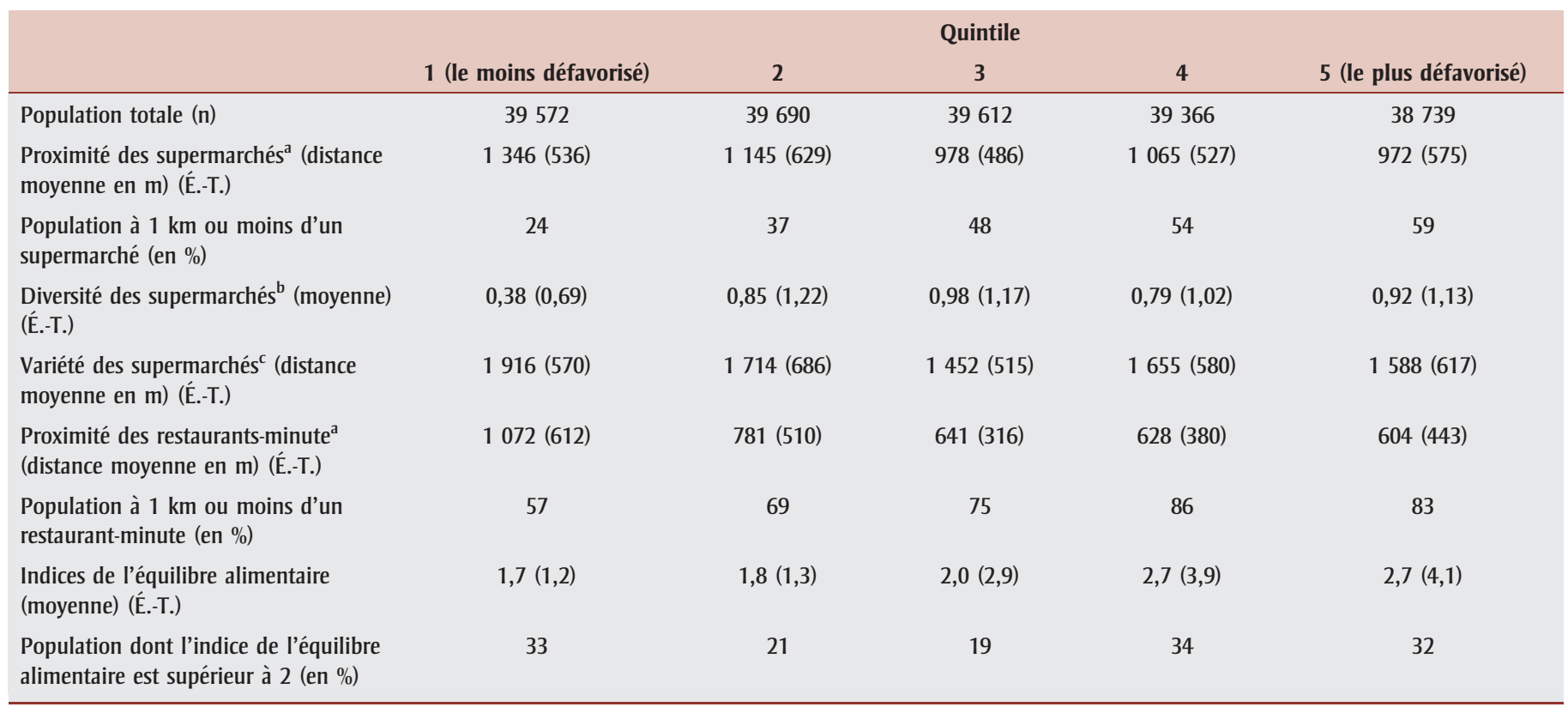

Abréviation : É.-T., écart-type.

a La proximité est définie comme étant la distance euclidienne (c.-à-d. en ligne droite) entre le centroïde (centre géographique) de chaque îlot urbain et chaque supermarché.

b La diversité est définie comme étant le nombre moyen de supermarchés à $1 \mathrm{~km}$ ou moins du centroïde de chaque îlot.

c La variété est définie comme étant la distance moyenne entre le centroïde de chaque îlot et les trois supermarchés de chaînes différentes les plus proches. 
FIGURE 4

Diversité des supermarchés, Saskatoon (Saskatchewan)

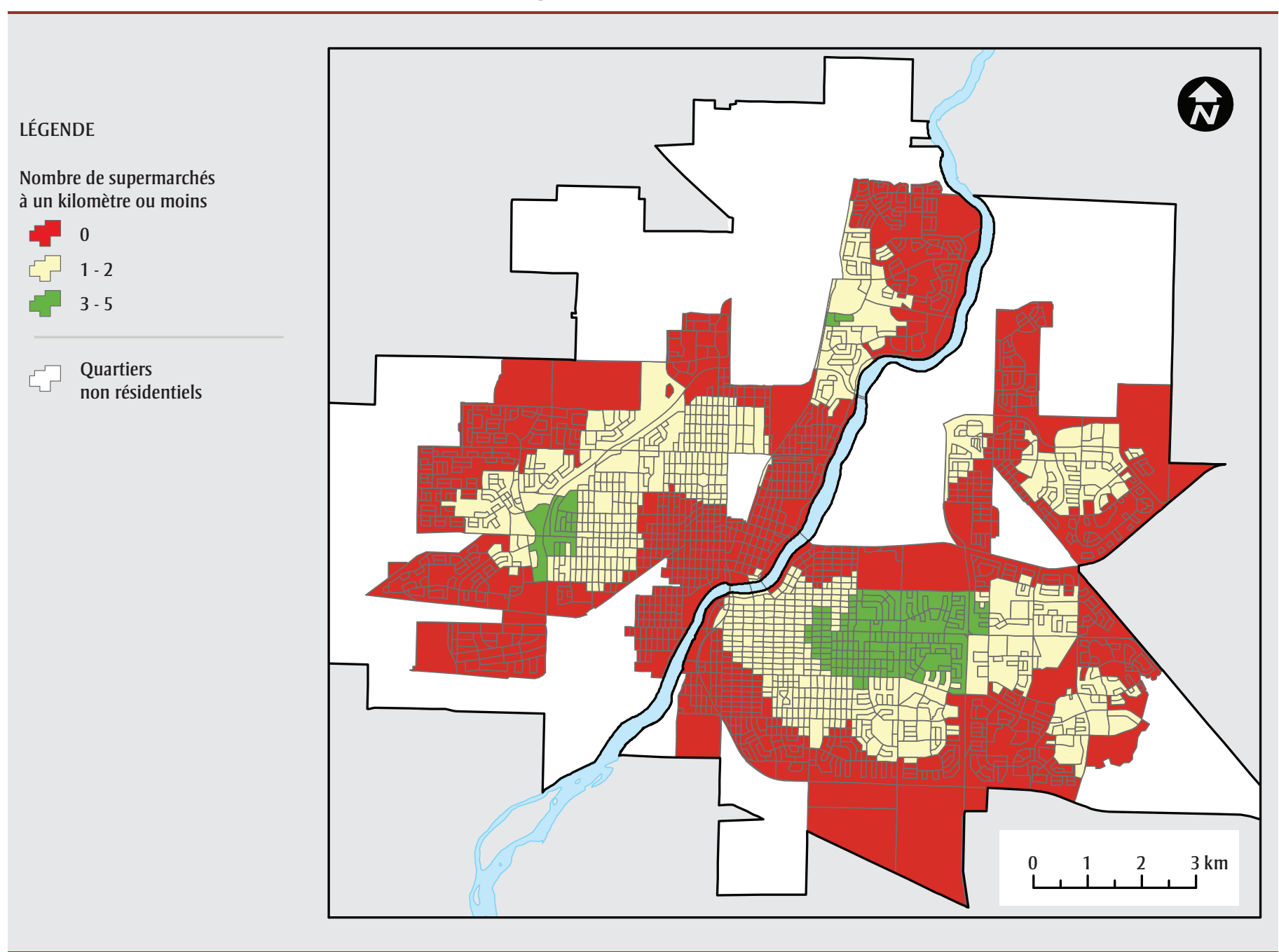

deuxième quintile étaient significativement plus éloignés des trois supermarchés les plus proches que les îlots du premier quintile (tableau 1). Nous n'avons pas dégagé de tendance claire entre variété et défavorisation matérielle.

Lorsque nous examinons la variété en fonction de la défavorisation sociale, nous constatons que les îlots les moins défavorisés (premier quintile) sont beaucoup plus loin des trois supermarchés les plus proches que les îlots des autres quintiles (tableau 2). La encore, nous n'avons pas observé de tendance claire à ce titre pour ce qui est de la défavorisation sociale.

\section{Restaurants-minute}

\section{Proximité}

Les trois quarts (74\%) des résidents de la ville vivaient à $1 \mathrm{~km}$ ou moins d'un restaurant-minute, et la distance moyenne pour se rendre au restaurant-minute le plus proche était de 724 mètres (figure 6). De manière générale, les îlots les plus éloignés des restaurants-minute se trouvaient en périphérie de la ville, où le niveau de défavorisation était mixte.

La proximité du restaurant-minute le plus proche variait considérablement en fonction de la défavorisation matérielle. Les îlots les plus défavorisés (cinquième quintile) étaient beaucoup plus proches des restaurants-minute les plus proches que les îlots les moins défavorisés (premier quintile). Cependant, la proximité avec le restaurant-minute le plus proche ne semble pas correspondre à un gradient socioéconomique (tableau 1).

Quant à la défavorisation sociale, les îlots les moins défavorisés (premier quintile) étaient situés beaucoup plus loin du restaurant-minute le plus proche que les îlots des autres quintiles (tableau 2). La proximité avec le restaurant-minute le plus proche était souvent plus grande 
FIGURE 5

Variété des supermarchés, Saskatoon (Saskatchewan)

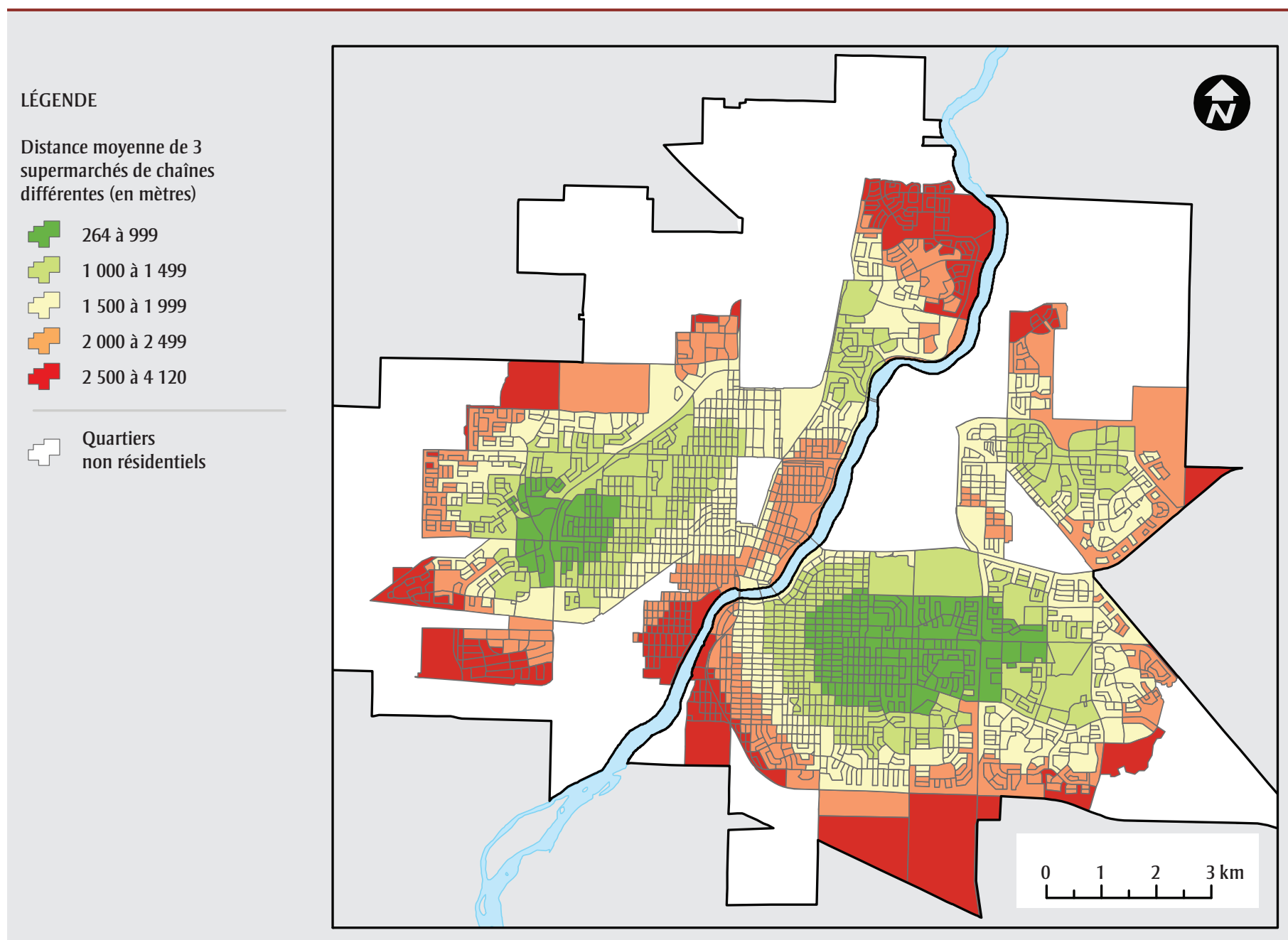

lorsque la défavorisation sociale était plus grande.

\section{Équilibre alimentaire}

Le ratio moyen de l'équilibre alimentaire à Saskatoon est de 2,3, ce qui signifie que le résident moyen habite plus de deux fois plus loin d'un supermarché que d'un restaurant-minute (figure 7 ). Cependant, certains secteurs de l'ouest de la ville, plus défavorisés sur les plans matériel et social, affichaient un ratio d'équilibre alimentaire s'élevant à 58,8.

L'équilibre alimentaire variait considérablement en fonction de la défavorisation matérielle. Certains des îlots les plus défavorisés (quatrième et cinquième quintiles) étaient beaucoup moins équilibrés que les îlots les moins défavorisés (premier et deuxième quintiles) (tableau 1). L'équilibre alimentaire se détériorait généralement lorsque la défavorisation matérielle était plus importante.

L'équilibre alimentaire variait également considérablement en fonction de la défavorisation sociale (tableau 2). Certains îlots des quatrième et cinquième quintiles affichaient un déséquilibre beaucoup plus prononcé que ceux des premier et troisième quintiles. L'équilibre alimentaire diminuait lorsque la défavorisation sociale augmentait.

\section{Secteurs préoccupants}

Nous avons combiné les résultats de l'indice d'équilibre alimentaire à ceux de l'indice d'accessibilité afin de cerner les secteurs préoccupants dans les quartiers de Saskatoon les plus défavorisés sur le plan matériel ou social (cinquième quintile). La figure 8 présente ces secteurs en fonction de la défavorisation matérielle, la figure 9 en fonction de la défavorisation sociale. Sur le plan de la défavorisation matérielle, le secteur de Saskatoon concerné le plus étendu est situé au sud de la ville, à l'ouest de la rivière Saskatchewan Sud. Sur le plan de la défavorisation sociale, le secteur le plus étendu se situe plutôt au nord de la rivière.

\section{Analyse}

Nos résultats indiquent que l'accès aux supermarchés mesuré par la proximité, la diversité et la variété ne suit pas de schéma précis. La proximité est plus faible de manière générale lorsque la défavorisation matérielle est plus élevée, mais elle 
FIGURE 6

Proximité des restaurants-minute, Saskatoon (Saskatchewan)

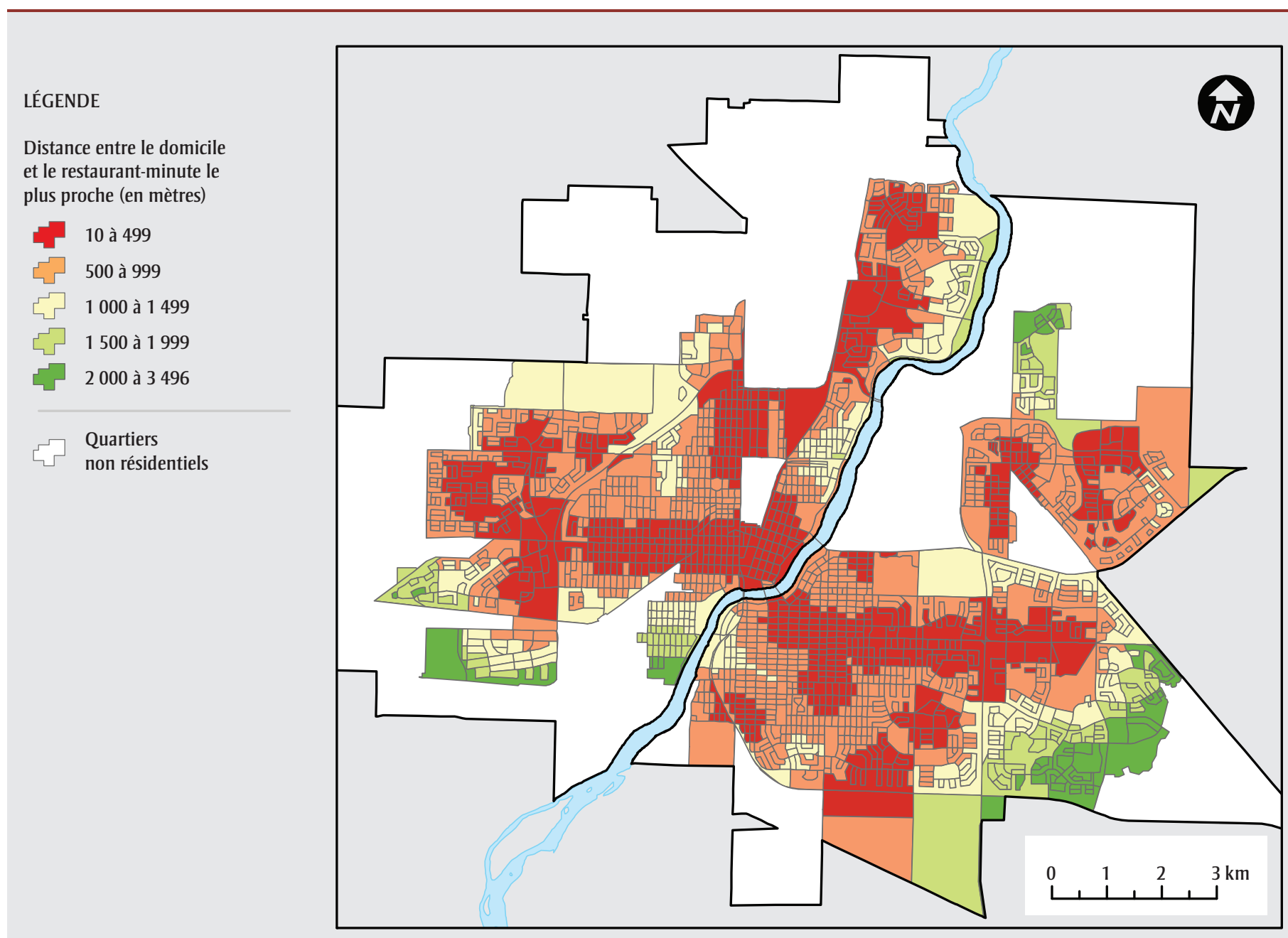

s'améliore lorsque la défavorisation sociale augmente. De même, la diversité est plus faible de manière générale lorsque la défavorisation matérielle est plus importante, quoique les îlots du deuxième quintile ne suivent pas ce schéma. Nous n’avons pas observé de schéma clair quant à la diversité en lien avec la défavorisation sociale ou à la variété en lien avec la défavorisation matérielle ou sociale. D’autres études canadiennes portant sur l'accès aux aliments et le statut socioéconomique en fonction de l'emplacement géographique n’ont pas, elles non plus, permis de dégager de schéma clair $^{7,10,12,41}$.

Les trois quarts des résidents de Saskatoon vivent à une distance de marche (1 km ou moins) d'un restaurant-minute, mais moins de la moitié d'entre eux vivent à une distance de marche d'un supermarché. Cela peut être attribuable au grand écart entre le nombre de supermarchés $(n=22)$ et le nombre de restaurants-minute $(n=120)$ évalués dans l'étude. Cependant, cela n'explique toujours pas pourquoi certains secteurs plus défavorisés à l'ouest présentaient un ratio d'équilibre alimentaire aussi élevé $(58,8)$. La proximité des restaurants-minute ne suit pas un schéma précis en fonction de la défavorisation matérielle, mais, en général, elle augmente proportionnellement à la défavorisation sociale.

Les secteurs préoccupants ne sont pas les mêmes selon le point de vue de la défavorisation sociale et celui de la défavorisation matérielle. Les études antérieures ont presque toutes exclusivement tenu compte du lien entre l'accès aux aliments et la défavorisation matérielle. Il est cependant très plausible que la défavorisation sociale constitue un élément distinct qui exige un examen plus approfondi. Des études à l'échelon individuel devraient être menées afin de déterminer de quelle façon la défavorisation matérielle et sociale dans chaque secteur a des répercussions sur l'accès aux aliments et, en fin de compte, sur les comportements liés à la santé. En d'autres termes, comment l'accès aux aliments et la défavorisation selon le secteur ont-ils des répercussions sur le type d'aliments achetés et consommés? Les recherches les liens entre santé et localisation doivent tenir compte de manière plus complète des mécanismes reliant les caractéristiques du bâti et les comportements individuels liés à la santé comme la consommation d'aliments sains ${ }^{13,27}$. 
FIGURE 7

Ratio de l'équilibre alimentaire, Saskatoon (Saskatchewan)

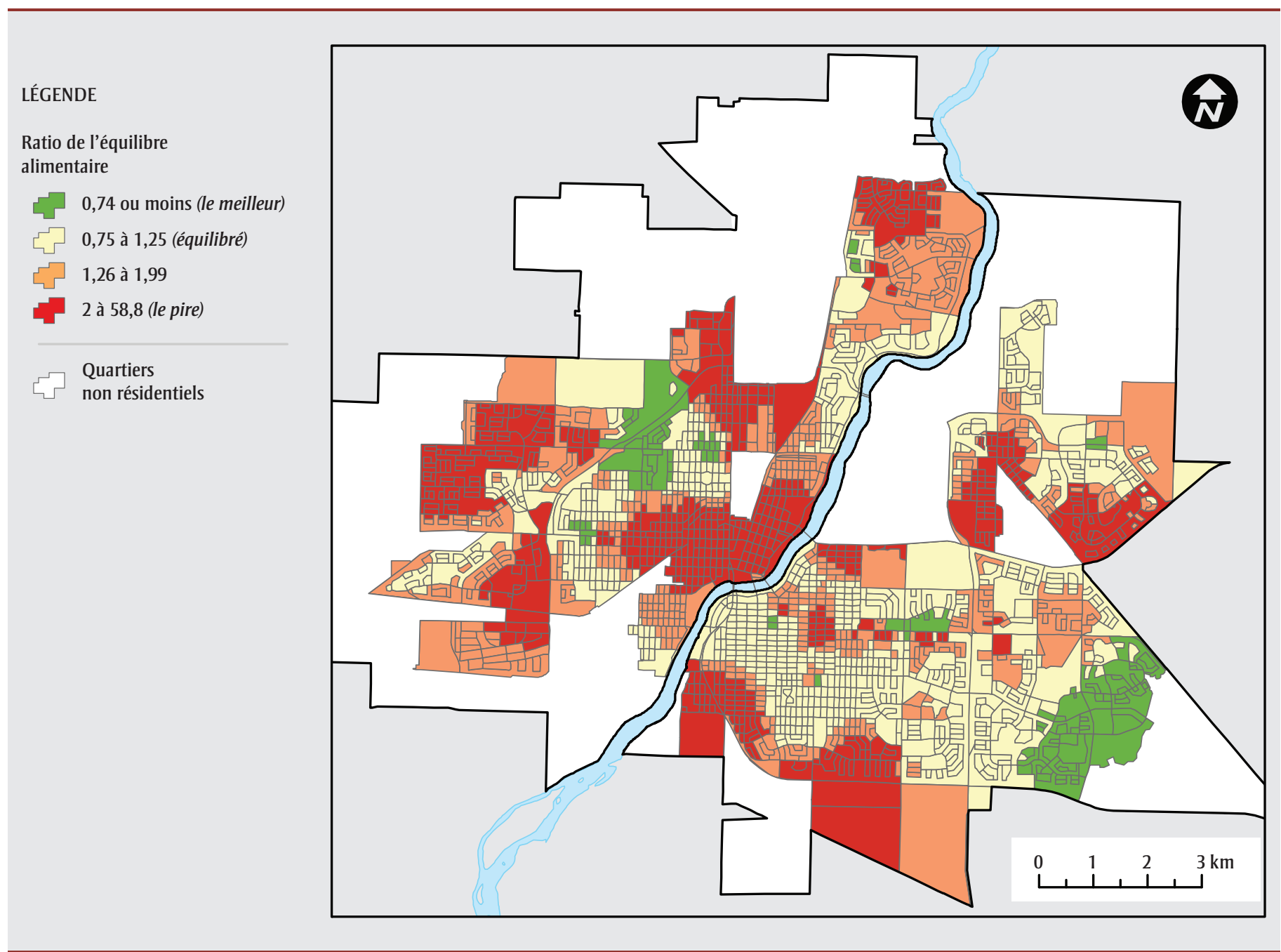

En ce qui a trait à la méthodologie, notre étude est une contribution originale à la littérature sur le sujet. La majorité des études sur l'accès aux aliments n'ont eu recours qu'à une seule mesure de l'accès ${ }^{14,46,47}$. Certaines d'entre elles ont bien eu recours à plus d'une mesure de l'accès ${ }^{7,48}$, mais très peu ont combiné un indice d'accessibilité et un indice d'équilibre alimentaire ${ }^{23}$. Notre indice d'équilibre alimentaire était similaire à l’indice de l'environnement des magasins d'alimentation (Retail Food Environment Index) utilisé dans une étude menée à Edmonton (Alberta), qui a additionné le nombre de restaurants-minute et le nombre de dépanneurs puis a divisé leur somme par le nombre d'épiceries et d'épiceries fines se trouvant à l'intérieur d'un périmètre donné ${ }^{29}$. Si nous avions appliqué cet indice à Saskatoon, il est probable que les indices de déséquilibre alimentaire auraient été encore plus élevés, car les dépanneurs y sont beaucoup plus nombreux que les épiceries fines.

Une autre contribution originale de notre étude a été l'utilisation d'un indice de défavorisation qui s'applique aux dimensions matérielle et sociale. Comme nous l'avons déjà mentionné, l'accès aux aliments a d'abord et avant tout été étudié en rapport avec la défavorisation matérielle, les répercussions de la défavorisation sociale ayant été peu étudiées. Étant donné que l'indice de défavorisation de l'INSPQ a servi à évaluer l'état de santé et les résultats sur la santé dans des quartiers urbains des principales métropoles au Canada $^{49}$, notre méthodologie pourrait facilement être appliquée à d'autres villes canadiennes en vue de faciliter les comparaisons entre les provinces et territoires en utilisant les seuils nationaux (pour notre étude, ce sont les seuils locaux qui ont été utilisés).

\section{Limites}

Bien que, dans le cadre de notre étude, nous ayons adopté des approches novatrices pour déterminer les liens entre l'accès aux aliments, l'équilibre alimentaire et la défavorisation, il convient de relever certaines limites. Tout d'abord, même si nous avons tenu compte de bon nombre de dimensions de l'accès, nous n'avons pas tenu compte de l'accès économique. Par exemple, le prix des aliments moins sains est généralement plus bas ${ }^{50}$. Les études à venir devraient tenir compte du lien entre l'accès et le prix 
FIGURE 8

Zones géographiques matériellement défavorisées ayant un pauvre accès aux supermarchés et un pauvre équilibre alimentaire, Saskatoon (Saskatchewan)

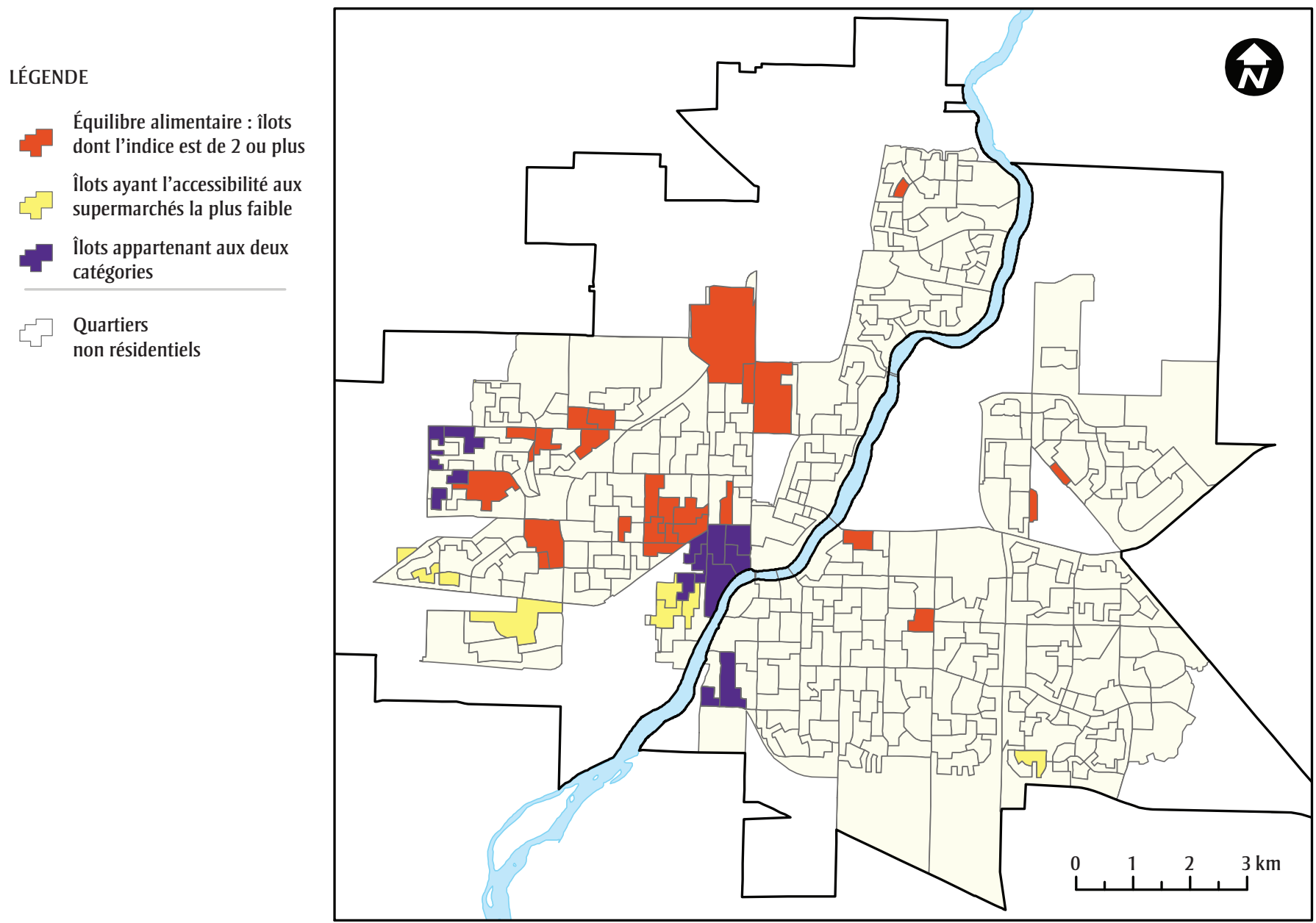

des aliments ainsi que de la manière dont ceux-ci sont en lien avec la défavorisation du quartier et avec les schémas de consommation sur le plan individuel.

Dans le cadre de notre étude, nous n'avons pas tenu compte de la mobilité. En d'autres termes, la population n'a pas toujours accès à des aliments dans le secteur où elle vit ${ }^{27}$. Par exemple, selon une étude récente menée à Detroit (ÉtatsUnis), l'espace d'activité (c.-à-d. l'espace où l'on mène ses activités quotidiennes) variait souvent en fonction du SSE ${ }^{51}$.

Une autre limite de notre étude était sa nature écologique. L'accès dans le secteur ne garantit pas que toutes les personnes qui y vivent fréquentent les mêmes magasins d'alimentation ou, plus impor- tant encore, consomment les mêmes aliments. Comme nous l'avons déjà mentionné, les prochaines études devraient tenir compte du lien entre les mesures en fonction du secteur, telles que l'accès aux aliments, et les mesures individuelles, telles que les habitudes alimentaires et les résultats sur la santé. Les habitudes alimentaires individuelles serviraient à déterminer si les personnes choisissent des aliments sains au supermarché ou des aliments malsains dans des restaurants-minute. Il serait important de se pencher sur cette question, car certains restaurants-minute tentent d'offrir des options plus saines.

Dans le cadre de notre étude, nous n'avons pas tenu compte des tendances temporelles. Bien que certaines de nos données proviennent d'années différentes (2006-2008), notre étude était de nature transversale. Les études similaires étaient presque entièrement de nature transversale, mais le lien entre l'environnement et la santé évolue par nature avec le temps ${ }^{52}$. Les études longitudinales devraient constituer une autre innovation dans ce secteur de recherche, intégrant une mesure et une cartographie de l'accès à différents moments.

Enfin, les études futures devraient élargir les critères d'inclusion en ce qui concerne les magasins d'alimentation. Nous n'avons pas tenu compte des petits supermarchés indépendants dans la présente analyse, car ils vendent habituellement des aliments plus chers et offrent une variété moindre que les supermarchés de 
FIGURE 9

Zones géographiques socialement défavorisées ayant un pauvre accès aux supermarchés et un pauvre équilibre alimentaire, Saskatoon (Saskatchewan)

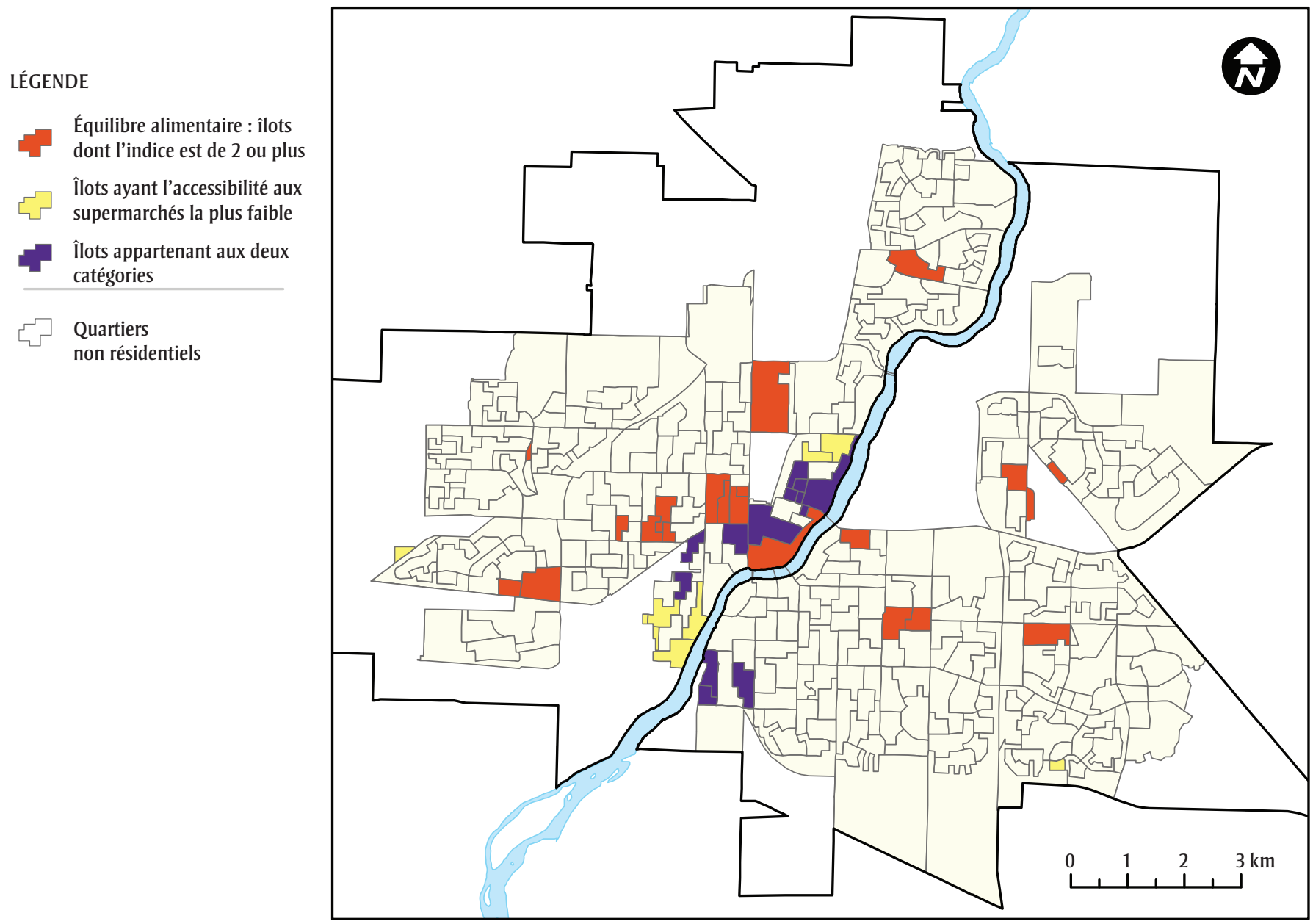

plus grande taille. Cependant, à certains endroits, ils constituent la source d'aliments sains la plus proche. De plus, même si nous avons tenu compte des dépanneurs qui vendent des repas chauds, on pourrait inclure tous les dépanneurs dans les prochaines études étant donné les résultats obtenus dans d'autres études. Par exemple, dans le cadre d'une étude américaine, les chercheurs ont conclu que plus il y a de dépanneurs dans un secteur, plus il est probable que les indices de masse corporelle soient plus élevés ${ }^{53}$. Dans d'autres études, les chercheurs ont également tenu compte de restaurants autres que les restaurants-minute ${ }^{6}$.

Malgré ses limites, l'étude donne à penser qu'il faut prévoir des interventions sur le plan des politiques et des programmes afin d'améliorer l'accès aux aliments dans la ville, surtout dans les secteurs défavorisés à l'ouest de la rivière Saskatchewan Sud. L'une des interventions visant les secteurs préoccupants cernés dans l'étude est le centre communautaire Station 20 West situé au sud-ouest de la rivière Saskatchewan Sud. Le centre offrira une épicerie sans but lucratif proposant des aliments sains et abordables. D’autres interventions seraient possibles : inclure des magasins d'alimentation de qualité dans les critères de planification de chaque nouveau quartier aménagé dans la ville $^{54}$; offrir des incitatifs fiscaux relativement à la prestation de services d'alimentation dans les secteurs défavorisés ${ }^{54}$; mettre sur pied des marchés de produits frais dans les quartiers ${ }^{54}$; encourager l'agriculture urbaine ${ }^{55}$; offrir des services de transport spécialisé pour se rendre aux supermarchés ou des programmes de livraison par les supermarchés ${ }^{9,10}$. L'évaluation des interventions est une question clé à approfondir. Bien qu'un bon nombre d'interventions relatives à l'accès aux aliments et à l'équilibre alimentaire au Canada aient été proposées, peu de travaux de recherche ont été menés sur la mise en œuvre et l'efficacité de ces interventions ${ }^{29}$.

\section{Conclusion}

L'étude nous permet de conclure que, à Saskatoon, l'accès aux aliments et l'équilibre alimentaire variaient en fonction de la défavorisation matérielle et sociale. Certaines mesures de l'accès aux aliments semblaient suivre un gradient socioécono- 
mique (p. ex. la proximité des supermarchés en lien avec la défavorisation matérielle; l'équilibre alimentaire diminuant à mesure que la défavorisation matérielle et sociale augmentait). Cependant, d'autres mesures ne suivaient pas de schéma clair. Ces résultats mettent en évidence l'importance des études contextuelles sur l'accès aux aliments, car différents schémas ont été observés d’un pays et d'une ville à l'autre ainsi que dans un même pays ou une même ville. L'étude a également mis en lumière bon nombre d'autres thèmes qui devraient être explorés dans le contexte de Saskatoon, tels que les habitudes alimentaires individuelles, la mobilité, la dimension temporelle de l'accès aux aliments et l'accès économique.

\section{Références}

1. Glanz K, Sallis JF, Saelens B, Frank LD. Nutrition Environment Measures Survey in stores (NEMS-S) : development and evaluation. Am J Prev Med. 2007;32(4):282-9.

2. Rundle A, Neckerman KM, Freeman L, et al. Neighborhood food environment and walkability predict obesity in New York City. Environ Health Perspect. 2009;117(3):442-7.

3. Lovasi GS, Hutson MA, Guerra M, Neckerman KM. Built environments and obesity in disadvantaged populations. Epidemiol Rev. 2009;31:7-20.

4. Giskes K, van Lenthe F, Avendano-Pabon $\mathrm{M}$, Brug J. A systematic review of environmental factors and obesogenic dietary intakes among adults : are we getting closer to understanding obesogenic environments? Obes Rev. 2011;12:e95-106.

5. Charreire H, Casey R, Salze P, et al. Measuring the food environment using geographical information systems : a methodological review. Public Health Nutr. 2010;13(11):1773-85.

6. Larson NI, Story MT, Nelson MC. Neighbourhood environments : disparities in access to health foods in the U.S. Am J Prev Med. 2009;36(1):74-81.
7. Apparicio P, Cloutier MS, Shearmur R. The case of Montreal's missing food deserts : evaluation of accessibility to food supermarkets. Int J Health Geogr. 2007;6:4.

8. Burns $\mathrm{CM}$, Inglis AD. Measuring food access in Melbourne : access to healthy and fast foods by car, bus and foot in an urban municipality in Melbourne. Health Place. 2007;13:877-85.

9. Cassady D, Mohan V. Doing well by doing good? A supermarket shuttle feasibility study. J Nutr Educ Behav. 2004;36:67-70.

10. Smoyer-Tomic KE, Spence JC, Armhein C. Food deserts in the prairies? Supermarket accessibility and neighbourhood need in Edmonton, Canada. Prof Geogr. 2006;58(3):307-26.

11. Chung C, Myers S. Do the poor pay more for food? An analysis of grocery store availability and food price disparities. J Consum Aff. 1999;33(2):276-96.

12. Black JL, Carpiano RM, Fleming S, Lauster N. Exploring the distribution of food stores in British Columbia : associations with neighbourhood socio-demographic factors and urban form. Health Place. 2011;17:96170 .

13. Walker RE, Keane CR, Burke JG. Disparities and access to healthy food in the United States : a review of food deserts literature. Health Place. 2010;16:876-84.

14. Morland K, Wing S, Diez Roux A. The contextual effect of the local food environment on residents' diets : the atherosclerosis risk in communities study. Am J Public Health. 2002;92(11):1761-7.

15. Odoms-Young AM, Zenk S, Mason M. Measuring food availability and access in African-American communities : implications for intervention and policy. Am J Prev Med. 2009:36(4 Suppl):S145-50.

16. Sallis JF, Glanz K. Physical activity and food environments : solutions to the obesity epidemic. Milbank Q. 2009;87(1):123-54.

17. Zenk SN, Lachance LL, Schulz AJ, Mentz G, Kannan S, Ridella W. Neighborhood retail food environment and fruit and vegetable intake in a multiethnic urban population. Am J Health Promot. 2009;23(4):255-64.
18. Moore LV, Diez Roux AV, Nettleton JA, Jacobs DR Jr. Associations of the local food environment with diet quality-a comparison of assessments based on surveys and geographic information systems : the multiethnic study of atherosclerosis. Am J Epidemiol. 2008;167(8):917-24.

19. Diez Roux AV, Mair C. Neighborhoods and health. Ann N Y Acad Sci. 2010;1186:12545 .

20. Black J, Macinko J. Neighborhoods and obesity. Nutrition Rev. 2008;66(1):2-20.

21. Davis B, Carpenter C. Proximity of fast-food restaurants to schools and adolescent obesity. Am J Public Health. 2009;99(3):50510.

22. Maddock J. The relationship between obesity and the prevalence of fast food restaurants : state-level analysis. Am J Health Promot. 2004;19(2):137-43.

23. Mari Gallagher Research and Consulting Group. Good food : examining the impact of food deserts on public health in Chicago. Chicago (IL): Mari Gallagher Research and Consulting Group; 2006.

24. Hemphill E, Raine K, Spence J, SmoyerTomic KE. Exploring obesogenic food environments in Edmonton, Canada : the association between socioeconomic factors and fast food outlets. Am J Health Promot. 2008;22(6):426-32 .

25. Powell LM, Auld MC, Chaloupka FJ, O'Malley PM, Johnston LD. Access to fast food and food prices : relationship with fruit and vegetable consumption and overweight among adolescents. Adv Health Econ Health Serv Res. 2006;17:23-48.

26. Sturm R, Datar A. Body mass index in elementary school children, metropolitan area food prices and food outlet density. Public Health. 2005;119:1059-68.

27. Feng J, Glass TA, Curriero FC, Stewart WF, Schwartz BS. The built environment and obesity : a systematic review of the epidemiologic evidence. Health Place. 2010;16:175-90.

28. Mari Gallagher Research \& Consulting Group. Examining the impact of food deserts on public health in Detroit. Chicago (IL): Mari Gallagher Research \& Consulting; 2007. 
29. Spence JC, Cutumisu N, Edwards J, Raine KD, Smoyer-Tomic K. Relation between local food environments and obesity among adults. BMC Public Health. 2009;9:192.

30. Power EM. Les déterminants de la saine alimentation chez les Canadiens à faible revenu. Revue canadienne de santé publique. 2005;96:S42-48.

31. Smith DM, Cummins S, Taylor M, et al. Neighbourhood food environment and area deprivation : spatial accessibility to grocery stores selling fresh fruit and vegetables in urban and rural settings. Int J Epidemiol. 2010;39(1):277-84.

32. Winkler E, Turrell G, Patterson C. Does living in a disadvantaged area mean fewer opportunities to purchase fresh fruit and vegetables in the area? Findings from the Brisbane food study. Health Place. 2006;12:306-19.

33. Woods F. Access to food in Saskatoon's core neighborhood. Saskatoon (SK): Community-University Institute for Social Research; 2003.

34. Beaulac J, Kristjansson E, Cummins S. A systematic review of food deserts, 19662007. Prev Chronic Dis. 2009;6(3): 1-10.

35. Cummins S, Macintyre S. The location of food stores in urban areas : a case study in Glasgow. Brit Food J. 1999;101(7):545-53.

36. Economic Research Service. Overview : food desert locator [Internet]. (ERS), U.S. Department of Agriculture (USDA) [Mis à jour le 6 juillet 2012; cité le 5 novembre 2012]. Consultable en ligne à la page : http ://www.ers.usda.gov/data-products/fooddesert-locator.aspx

37. Smoyer-Tomic K, Spence J, Raine K, et al. The association between neighborhood socioeconomic status and exposure to supermarkets and fast food outlets. Health Place. 2008;14:740-54.

38. Block J, Scribner R, DeSalvo K. Fast food, race/ethnicity, and income : a geographic analysis. Am J Prev Med. 2004;27(3): 2117.

39. Pearce J, Blakely T, Witten K, Bartie P. Neighborhood deprivation and access to fast-food retailing : a national study. Am J Prev Med. 2007;32(5):375-82.
40. Fleischhacker SE, Evenson KR, Rodriguez DA, Ammerman AS. A systematic review of fast food access studies. Obes Rev. 2011;12:e460-71.

41. Larsen K, Gilliland J. Mapping the evolution of 'food deserts' in a Canadian city : supermarket accessibility in London, Ontario, 1961-2005. Int J Health Geogr. 2008;7:16.

42. Kaufman P, Lutz S. Competing forces affect food prices for low income households. Food Rev. 1997;20(2);8-12.

43. Austin SB, Melly SJ, Sanchez BN, Patel A, Buka S, Gortmaker SL. Clustering of fastfood restaurants around schools : a novel application of spatial statistics to the study of food environments. Am J Public Health. 2005;95(9):1575-81.

44. Pampalon R, Hamel D, Gamache P, Raymond G. Un indice de défavorisation pour la planification de la santé au Canada. Maladies chroniques au Canada. 2009;29 (4):199-213.

45. Statistique Canada. Cartes de référence des aires de diffusion, guide de référence année de recensement 2006. Ottawa (Ont.) : Statistique Canada; 2007. [n : 92-145-GIF au catalogue].

46. Cummins S, Macintyre S. The location of food stores in urban areas : a case study of Glasgow. Brit Food J. 1999;101(7):545-53.

47. Zenk SN, Schulz AJ, Israel BA, James SA, Bao S, Wilson ML. Neighborhood racial composition, neighborhood poverty, and the spatial accessibility of supermarkets in metropolitan Detroit. Am J Public Health. 2005;95(4):660-7.

48. Sharkey JR, Horel S, Han D, Huber JC. Association between neighbourhood need and spatial access to food stores and fast food restaurants in neighborhoods of Colonias. Int J Health Geogr. 2009;8(9): S151-5.

49. Initiative sur la santé de la population canadienne. Réduction des écarts en matière de santé : un regard sur le statut socioéconomique en milieu urbain au Canada. Ottawa (Ont.): ICIS; 2008.

50. Drewnowski A, Darmon N. Food choices and diet costs : an economic analysis. J Nutr. 2005;135(4):900-4.
51. Zenk SN, Schulz AJ, Matthews, SA, et al. Activity space environment and dietary and physical activity behaviours : a pilot study. Health Place. 2011;17:1150-61.

52. Cummins S, Macintyre S. Food environments and obesity-neighbourhood or nation? Int J Epidemiol. 2006;34:100-4.

53. Powell LM, Auld MC, Chaloupka FJ, O'Malley PM, Johnston LD. Associations between access to food stores and adolescent body mass index. Am J Prev Med. 2007;33(4 Suppl): S301-7.

54. Toronto Food Policy Council. Food retail access and food security for Toronto's lowincome citizens. Toronto (ON): Food Policy Council; 1996. Discussion paper series no.7. $32 \mathrm{p}$.

55. Desjardins E, Lubczynski J, Xuereb M. Incorporating policies for a healthy food system into land use planning : the case of Waterloo Region, Canada. JAFSCD. 2011;2(1):127-4 